\title{
Ciencia abierta y bibliotecas académicas: una revisión sistemática de la literatura
}

\section{Open science and academic library: a systematic literature review}

\section{Ciência aberta e bibliotecas acadêmicas: Uma revisão sistemática da literatura}

\author{
Jaider Ochoa-Gutiérrez \\ Escuela Interamericana de Bibliotecología \\ Universidad de Antioquia, Medellín-Colombia \\ jaider.ochoa@udea.edu.co \\ Alejandro Uribe-Tirado \\ Escuela Interamericana de Bibliotecología \\ Universidad de Antioquia, Medellín-Colombia \\ alejandro.uribe2@udea.edu.co
}

\begin{abstract}
Resumen: Este estudio presenta un estado de la cuestión sobre el papel de la biblioteca académica (universitaria o de investigación) en el desarrollo de la ciencia abierta a nivel mundial. A partir de este artículo, se da a conocer el estado de experiencias y desarrollos que se han dado en esta materia y así poder obtener un panorama general de los aportes de las bibliotecas académicas. Para su desarrollo se realizó una revisión sistemática de la literatura, a partir de diferentes fuentes de información, tanto abiertas como cerradas, y se realizó un análisis métrico y hermenéutico para la selección de los aportes más significativos y la elaboración de nuevos aportes teórico-conceptuales al tema. Los resultados muestran en general, la influencia directa de las bibliotecas académicas en el desarrollo de la ciencia abierta; sin embargo, exceptuando el acceso abierto, se presenta poco desarrollo de los diferentes componentes de la misma, mostrando la importancia de fortalecer un marco teórico-conceptual sobre la ciencia abierta y la identificación de estrategias, retos y perspectivas que oriente el fortalecimiento de las capacidades de las bibliotecas académicas. Este texto, además de la revisión sistemática misma, es un aporte para identificar casos y buenas prácticas para todas aquellas bibliotecas académicas de nuestro contexto latinoamericano que están previendo ser un soporte clave para la ciencia abierta en sus universidades, como se está requiriendo en la actualidad.
\end{abstract}

Palabras-clave: Ciencia abierta. Bibliotecas académicas. Bibliotecas universitarias. Bibliotecas de investigación. Universidad. Servicios para investigadores. Revisión sistemática.

Abstract: This study presents a state of the art on the role of the university library in the development of open science worldwide. From this article, the state of experiences and developments that have occurred in this subject is disclosed and thus to obtain a general overview of the contributions of the university libraries. For its development, a systematic literature review was conducted, from different sources of information, both open and closed, and a metric and hermeneutic analysis was carried out to select the most significant contributions and the preparation of new theoretical-conceptual contributions for the topic. The results show, in general, the direct influence of university libraries in the development of open science; however, except for open access, there is little development of the distinct components of the same, showing the importance of strengthening a theoretical-conceptual framework on open science and the identification of strategies, challenges and perspectives that guide the strengthening of capacities of university libraries. This text, in addition to the systematic review 
itself, is a contribution to identify cases and good practices for all those university libraries of our Latin American context that are foreseeing to be a key support for open science in their universities, as is currently required.

Keywords: Open Science. University library. Academic library. Research library. University. Research services. Systematic review.

Resumo: O estudo apresenta o estado da arte sobre o papel da biblioteca acadêmica (universitária ou de pesquisa) no desenvolvimento da ciência aberta no mundo. Divulgam-se as experiências e desenvolvimentos nesta temática e fornece-se uma visão geral das contribuições das bibliotecas acadêmicas. Foi realizada uma revisão sistemática da literatura a partir de diferentes fontes de informação, tanto abertas, quanto fechadas, bem como uma análise métrica e hermenêutica para a seleção das contribuições mais significativas e a elaboração de novas contribuições teóricas e conceituais sobre o assunto. Em geral, os resultados mostram a influência direta das bibliotecas acadêmicas no desenvolvimento da ciência aberta; no entanto, com exceção do acesso aberto, há pouco desenvolvimento dos outros componentes, mostrando a importância de fortalecer o referencial teórico-conceitual sobre ciência aberta e a identificação de estratégias, desafios e perspectivas que norteiem o fortalecimento das capacidades das bibliotecas acadêmicas. $\mathrm{O}$ trabalho, bem como a própria revisão sistemática, é uma contribuição para a identificação dos casos e das melhores práticas nas bibliotecas acadêmicas do contexto latino-americano que tem a intenção de se tornar um suporte importante da ciência aberta nas universidades.

Palavras-chave: Ciência aberta. Bibliotecas acadêmicas. Bibliotecas universitárias. Bibliotecas de pesquisa. Universidade. Serviços para pesquisadores. Revisão sistemática.

\section{Introducción}

La ciencia abierta es un movimiento internacional que ha cobrado mayor importancia en los últimos años. Como lo menciona Vicente-saez y Martinez-fuentes (2018).

\footnotetext{
Es un fenómeno disruptivo que está surgiendo alrededor del mundo y especialmente en Europa. La ciencia abierta produce cambios socioculturales y tecnológicos, basados en la apertura y la conectividad, y sobre cómo se diseña, ejecuta, captura y evalúa la investigación. las herramientas de datos abiertas, las plataformas de acceso abierto, los métodos de revisión abierta (open peer review) o las actividades de compromiso públicos (public engagement activities) son tendencias irreversibles, que están impactando a todos actores científicos y tienen el potencial de acelerar el ciclo de investigación.
}

En este sentido, países como Finlandia, Alemania, Holanda, Portugal y Gran Bretaña vienen liderando y avanzando en el desarrollo y la implementación de la ciencia abierta, principalmente a partir de políticas, infraestructuras y estrategias de sensibilización para su exitosa adopción (OCDE, 2015). 
Sin embargo, en los últimos años, se ha planteado la pregunta por el rol que juegan las bibliotecas académicas (universitarias o de investigación) ${ }^{1}$ en este contexto (ANGLADA; ABADAL, 2018), teniendo en cuenta el protagonismo que han tenido en el movimiento de acceso abierto, sobre todo en el desarrollo de la ruta verde (repositorios digitales) y ante la necesidad apremiante que presentan muchas instituciones frente al manejo de los datos abiertos y la cultura abierta.

Este estudio presenta un estado de la cuestión sobre el papel de la biblioteca académica en el desarrollo de la ciencia abierta a nivel mundial. Se da a conocer el estado de experiencias y desarrollos que se han dado en esta materia para lograr dar un panorama general de los aportes de esta tipología de bibliotecas.

Su desarrollo se realizó mediante una revisión sistemática de la literatura, teniendo en cuenta los diferentes parámetros que desde el Cochrane Handbook for Systematic Reviews of Interventions (HIGGINS; GREEN, 2011) son mencionados, y las recomendaciones que Salvador-Oliván, Marco-Cuenca y Arquero-Avilés (2018) indican, en este reciente trabajo, sobre revisiones sistemáticas desde nuestra área del conocimiento.

Inicialmente se realizó un análisis métrico que da cuenta de la generalidad de la producción, posteriormente se hace un análisis hermenéutico identificando los elementos más significativos y que permitan la elaboración de nuevos aportes teórico-conceptuales al tema.

Los resultados muestran en general, la influencia directa de las bibliotecas académicas en el desarrollo de la ciencia abierta; sin embargo, exceptuando el acceso abierto, se presenta aún poco desarrollo en todos los otros componentes de la ciencia abierta (Figura 1), mostrando la importancia de fortalecer un marco teórico-conceptual de la misma y la identificación de estrategias, retos y perspectivas que oriente el fortalecimiento de las capacidades de las bibliotecas académicas.

\footnotetext{
1 Para efectos de este texto, se asumirá al hablar de esta tipología de bibliotecas, como "bibliotecas académicas", en las cuales se incluyen las "bibliotecas universitarias" o "bibliotecas de investigación"- o centros de documentación-, ya que en la literatura se incluyen, la mayoría de las veces, sin diferenciación.
} 


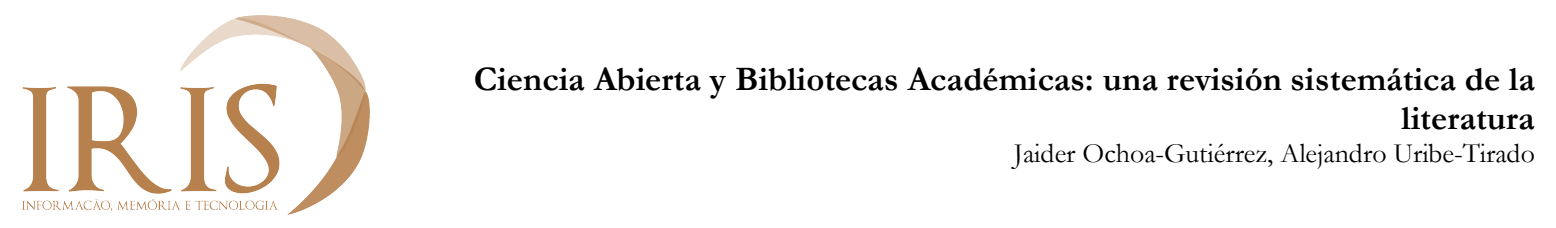

Figura 1. Principales componentes de la ciencia abierta

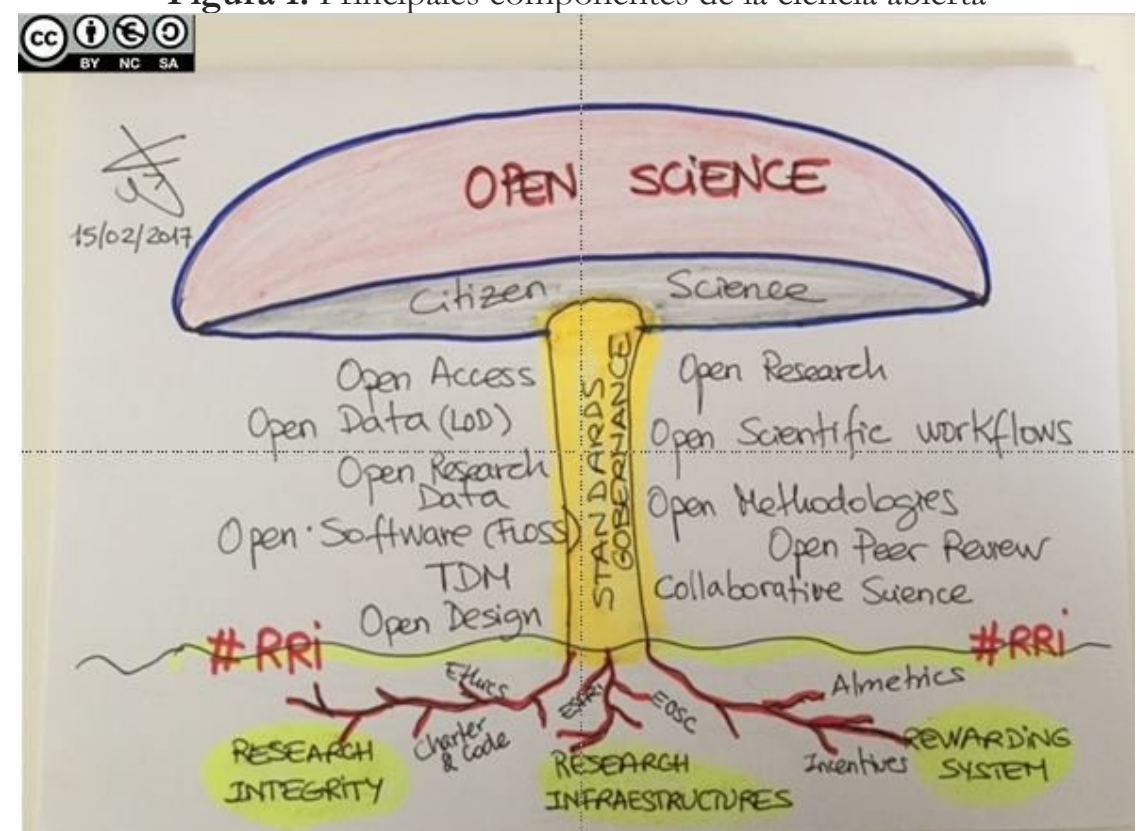

Fuente: Eva Mendéz: Open Science as an organism: https://pbs.twimg.com/media/C7cNfKmW4AESrGm.jpg

\section{Metodología}

Este trabajo presenta el estado de la cuestión que identifica conceptos, tendencias y experiencias de bibliotecas académicas en el marco del desarrollo de la ciencia abierta. La ruta metodológica se dio principalmente a partir de cuatro momentos:

- Selección de fuentes de información: Con el fin de garantizar una metodología eficiente de búsqueda, recuperación y depuración de la producción científica, además de incluir tanto fuentes cerradas como abiertas, por coherencia con el tema mismo ${ }^{2}$, se seleccionaron las siguientes:

- Bases de datos comerciales: Web of Science (WoS) y Scopus

- Repositorios y directorios: DOAJ, SciELO, Redalyc y E-LIS

- Redes sociales científicas: Research Gate

- Buscadores académicos: Google Scholar

\footnotetext{
${ }^{2}$ La mayoría de estudios métricos se centran casi siempre, en fuentes cerradas como WoS y SCOPUS, aunque para um panorama amplio, sería necesario incluir otras fuentes de preferencia abiertas.
} 
- Preguntas de investigación: Teniendo en cuenta el desarrollo metodológico propuesto por Pérez Rave (2012), este artículo busca responder dos preguntas: La primera, ¿Cuál es el rol de la biblioteca académica frente al desarrollo de la ciencia abierta? y la segunda, ¿Qué experiencias se han desarrollado en este marco?

- Recopilación de la producción cientifica: A partir de las fuentes seleccionadas, considerando la importancia de su variedad y de explicitar el proceso de búsqueda (HIGGINS Y GREEN, 2011; SALVADOR-OLIVÁN, MARCO-CUENCA, ARQUERO-AVILÉS, 2018), y acorde a las preguntas de investigación, se realizó la búsqueda -entre el 16 de junio y el 15 de julio de 2018- utilizando la ecuación: ("open science" OR "ciencia abierta" OR ciência aberta) AND (librar* OR biblioteca); principalmente en los campos de título o palabras clave, integrando diferentes opciones posibles de acceder a la información, para así analizar el estado y las tendencias de la producción científica. Respecto al período de tiempo contemplado, no se realizó una delimitación, ya que no se encontró un número elevado de documentos, y a su vez, se pretendió ubicar antecedentes respecto a la relación ciencia abierta-bibliotecas. No obstante, se puede evidenciar que la producción se concentra a partir de la primera década del 2000. Se logró recopilar 75 documentos entre artículos científicos, actas de congresos y reportes de instituciones o asociaciones bibliotecarias.

- Categorización y revisión de documentos: Se identificaron los documentos potenciales para la creación de un banco de datos que permitiera su depuración y gestión, a partir de este se eliminaron los textos duplicados, los que no fueron pertinentes para los objetivos de la investigación -otros tipos de bibliotecas - o aquellos en los que no fue posible acceder al texto completo. Una vez realizado este proceso, se consolidaron 47 documentos que fueron utilizados para el análisis ${ }^{3}$.

- Análisis de contenido: Después de realizar la depuración de los contenidos, se analizó el contenido mediante técnicas bibliométricas y hermenéuticas, y se extrajeron los aspectos más importantes respecto a la ciencia abierta de acuerdo con los siguientes elementos:

- Propuestas de relacionamiento de la ciencia abierta y bibliotecas académicas. 
- Casos de bibliotecas académicas que apoyan el desarrollo de la ciencia abierta.

- Reflexiones y retos de las bibliotecas académicas frente a la ciencia abierta.

\section{Resultados}

Según David (2004), la ciencia abierta trae consigo una gran revolución en la investigación científica, ya que permite la realización de análisis más transparentes e informes de estudios más abiertos y con posibilidades de intercambio de datos sin procesar. No obstante, hay una necesidad de posicionamiento y comprensión por parte de los investigadores para que esto pueda ser una realidad; asimismo, la preparación de infraestructuras, procesos de formación y metodologías abiertas que permitan su aprehensión por parte de las comunidades e instituciones de investigación.

Ante esto, Aibar (2013) dice que la mayoría de los expertos coinciden en que esta corriente está provocando modificaciones importantes en las prácticas de investigación y la publicación. Incluso Ogungbeni et al. (2016), argumentan que estas modificaciones reflejan una oferta concertada para aumentar la naturaleza colaborativa y la validez de los hallazgos publicados para tener una mejor comprensión de la ciencia y su disciplina asociada.

Sin embargo, este panorama requiere el compromiso de múltiples actores e instituciones, que aporten recursos y desarrollen estrategias para ir generando la cultura de la ciencia abierta, es decir, un ecosistema, donde uno de los actores presentes y claves, son las bibliotecas (SMITH; MARSAN, 2015; CROUZIER, 2015).

En este sentido, las bibliotecas se han convertido en una institución importante que contribuye a que el conocimiento y la información sean accesibles para la mayor cantidad de personas posibles. Por esto, para seguir siendo competitivos en un panorama científico cada vez más digitalizado y abierto, las bibliotecas académicas deberían monitorear permanentemente el contexto y desarrollar ofertas orientadas a la demanda a lo largo del ciclo de investigación (OGUNGBENI et al., 2016). Para ello, es importante la cooperación con otras bibliotecas y la articulación con otros estamentos que apoyan los procesos de investigación.

A partir de lo anterior, se presenta el análisis de la información recopilada, la cual da un panorama general de las propuestas y experiencias de la biblioteca académica como institución fundamental para el desarrollo de la ciencia abierta. Inicialmente se presenta un análisis métrico y posteriormente el análisis hermenéutico. 


\subsection{Análisis métrico}

\subsubsection{Producción por año}

Como se puede evidenciar en la Figura 2. La producción científica en el tema ha aumentado considerablemente en los últimos años, lo que tiene completa sincronía con los avances en torno al movimiento en general, vinculado principalmente con el desarrollo de políticas, programas y hojas de ruta en varios países del mundo.

Figura 2. Producción en ciencia abierta y bibliotecas académicas.

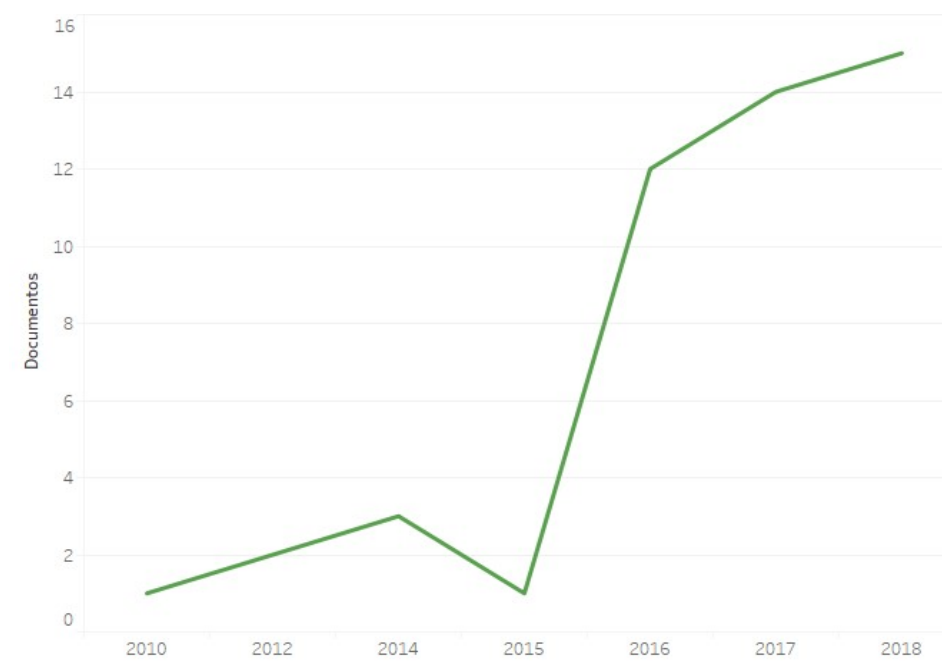

Fuente: Elaboración propia a partir de datos de Scopus, Web of Science, Google Scholar.

\subsubsection{Autores}

A partir de la Figura 3, principalmente se destacan dos autores: Paul Ayris, quien en sus publicaciones (AYRIS, 2017; AYRIS; IGNAT, 2018; AYRIS, et al, 2018; AYRIS, BERNAL, et al., 2018) habla de la importancia del rol de la biblioteca académicas y los retos en materia de crear y mantener políticas, infraestructuras, sistemas de publicación y servicios orientados a la ciencia abierta. Adicional a ello, hace hincapié en la importancia de la colaboración en Europa y de la importancia de proyectar un cambio cultural para la adopción de la ciencia abierta.

También se destaca Dallmeier-Tiessen (DALLMEIER-TIESSEN et al., 2012; DALLMEIER-TIESSEN \& MELE, 2014; CHEN et al., 2016), el cual ha centrado su investigación en la digital library of High-Energy Physicsen, aportando al desarrollo de bibliotecas digitales y de estrategias de preservación de datos para la investigación reproducible. 
Figura 3: Autores con mayor número de producción sobre el tema bibliotecas académicas y ciencia abierta.

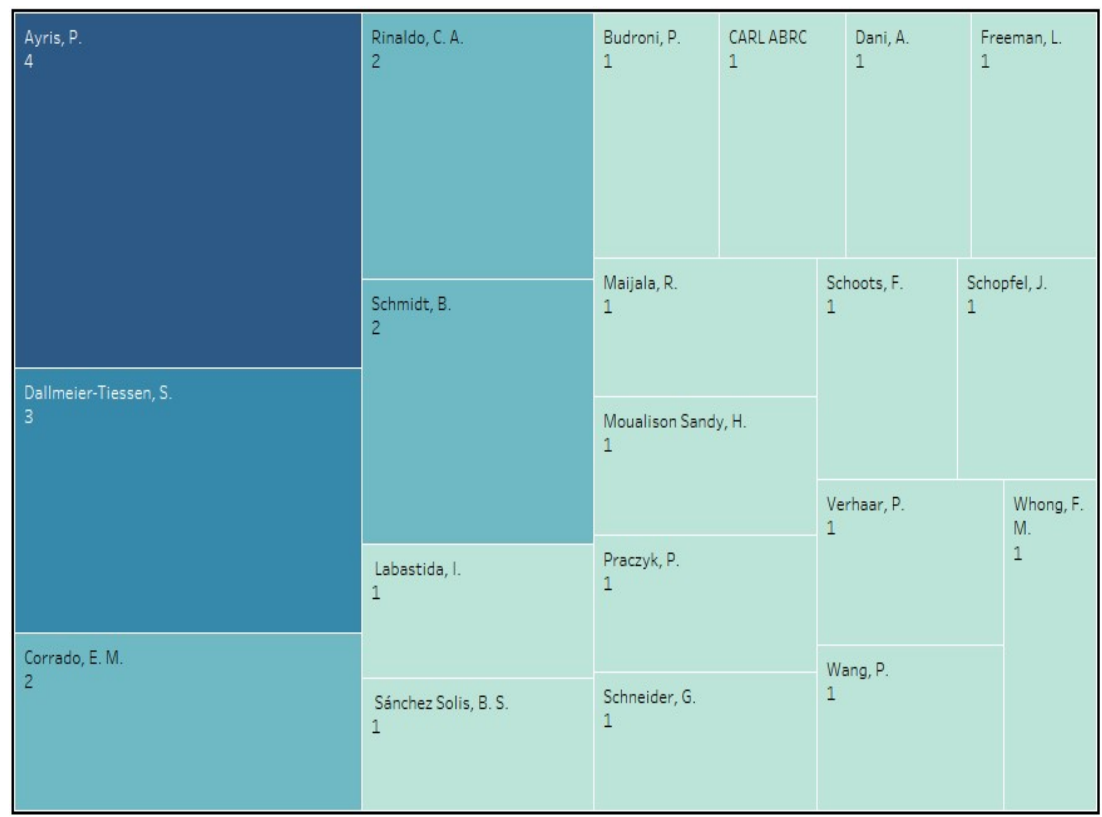

Fuente: Elaboración propia a partir de datos de Scopus, Web of Science, Google Scholar.

\subsubsection{Fuentes de publicación}

Respecto a las fuentes de publicación (Figura 4), se observa mayor número de publicaciones por parte de la revista LIBER Quartely, publicación en acceso abierto editada por The Association of European Research Libraries, y en la cual se abordan temas actuales en la investigación bibliotecológica y la gestión de información.

Figura 4. Fuentes de información con mayor número de producción sobre el tema bibliotecas académicas y ciencia abierta.

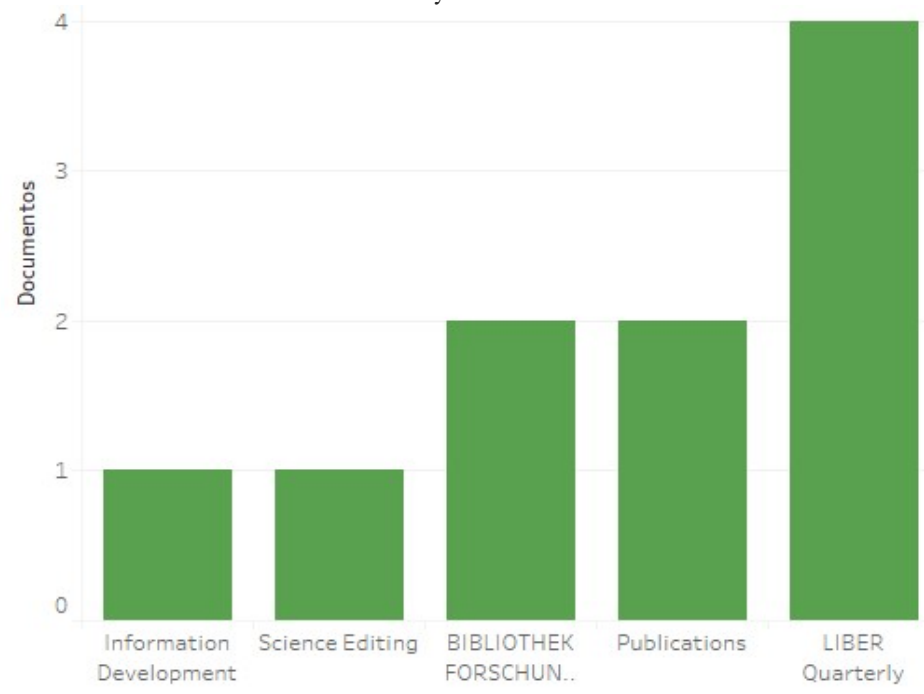

Fuente: Elaboración propia a partir de datos de Scopus, Web of Science, Google Scholar. 
Este hecho, que sea principalmente esta revista, no es de extrañar, ya que esta Asociación está haciendo una apuesta importante por asumir ese rol clave como bibliotecas, por impulsar la ciencia abierta, con planes continuos (https://libereurope.eu/strategy/) y donde se destacan varias estrategias (Figura 5):

Figura 5: Libraries Enabling Open Science: LIBER Strategy \& Advocacy.

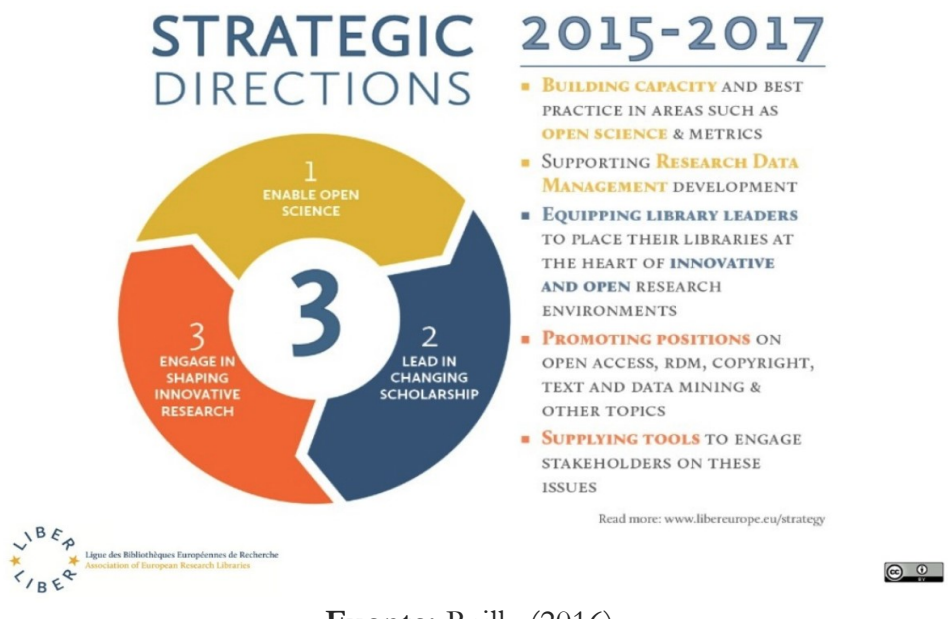

Fuente: Reilly (2016).

Y a su vez, destacar a diferentes bibliotecas de su Asociación, que están cumpliendo ese rol importante por la ciencia abierta (AYRIS et al, 2018):

- Karlsrube Institute of Technology (Alemania)

- National Library of Finland (Finlandia)

- Ruder Boškoović Institute Library (Croacia)

- Spanish National Research Council (España)

- Svetozar Markovic University Library (Serbia)

- University of Barcelona (España)

- University College London (Inglaterra)

- University Library of Southern Denmark (Dinamarca)

\subsubsection{Temas de investigación}




\section{IRIS)}

Ciencia Abierta y Bibliotecas Académicas: una revisión sistemática de la literatura Jaider Ochoa-Gutiérrez, Alejandro Uribe-Tirado

A partir del análisis de redes por co-ocurrencia de términos realizado en VOSViewer ${ }^{4}$ (Figura 6 y 7), se pueden identificar algunas temáticas de interés respecto al aporte de las bibliotecas académicas al desarrollo de la ciencia abierta.

Sustancialmente, se pueden ver varios clústeres de análisis:

- El primero, en color rojo, habla de la experiencia de las bibliotecas en el planteamiento y ejecución de proyectos o estrategias para la gestión de datos de investigación.

- El segundo, en amarillo, plantea los retos y el rol que juegan las bibliotecas académicas para la ciencia abierta, en sentido amplio.

- El tercero, en verde, muestra la recurrencia en la literatura de publicaciones que plantean la necesidad de orientar servicios y proyectos para el apoyo al desarrollo del ciclo de investigación.

- El cuarto y quinto (azul y morado), evidencian el desarrollo del open access y el apoyo a la publicación científica, como uno de los pilares fuertes de las bibliotecas académicas.

Figura 6: Análisis de redes de co-ocurrencia de términos.

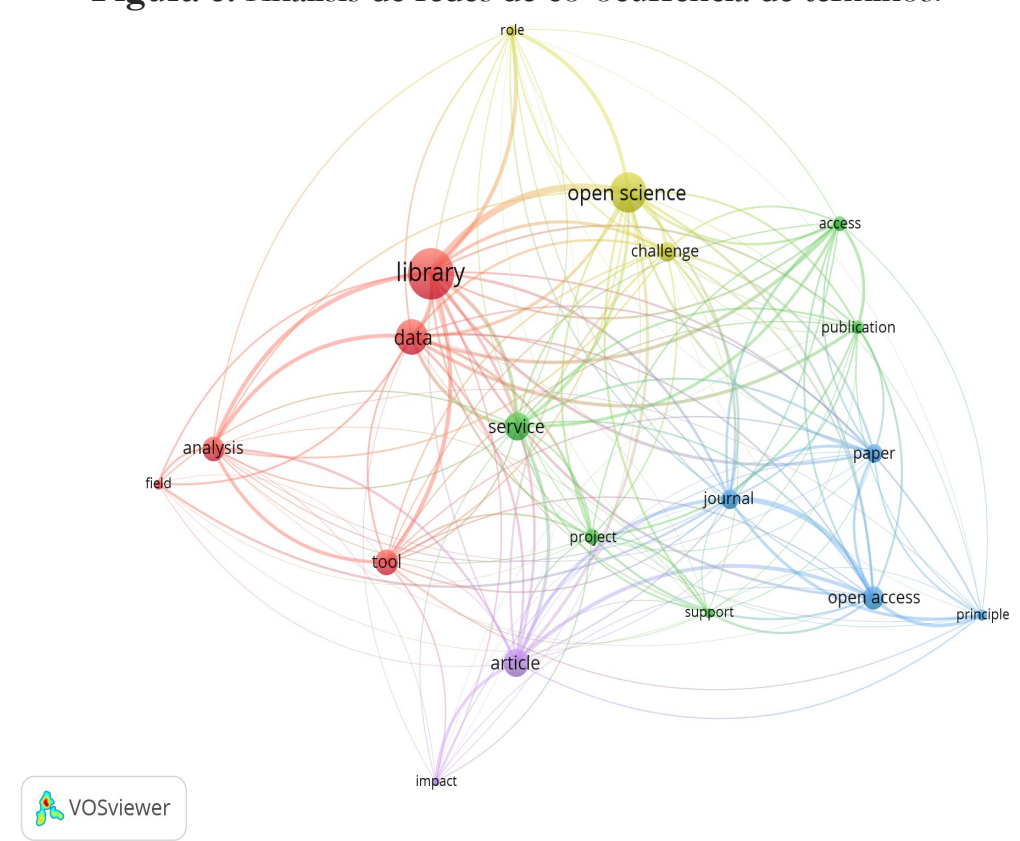

Fuente: VOSViewer

${ }^{4}$ http://www.vosviewer.com/ (Consultado: 15-7-2018) 
Figura 7: Análisis de redes de co-ocurrencia de términos. Relaciones directas con el término biblioteca.

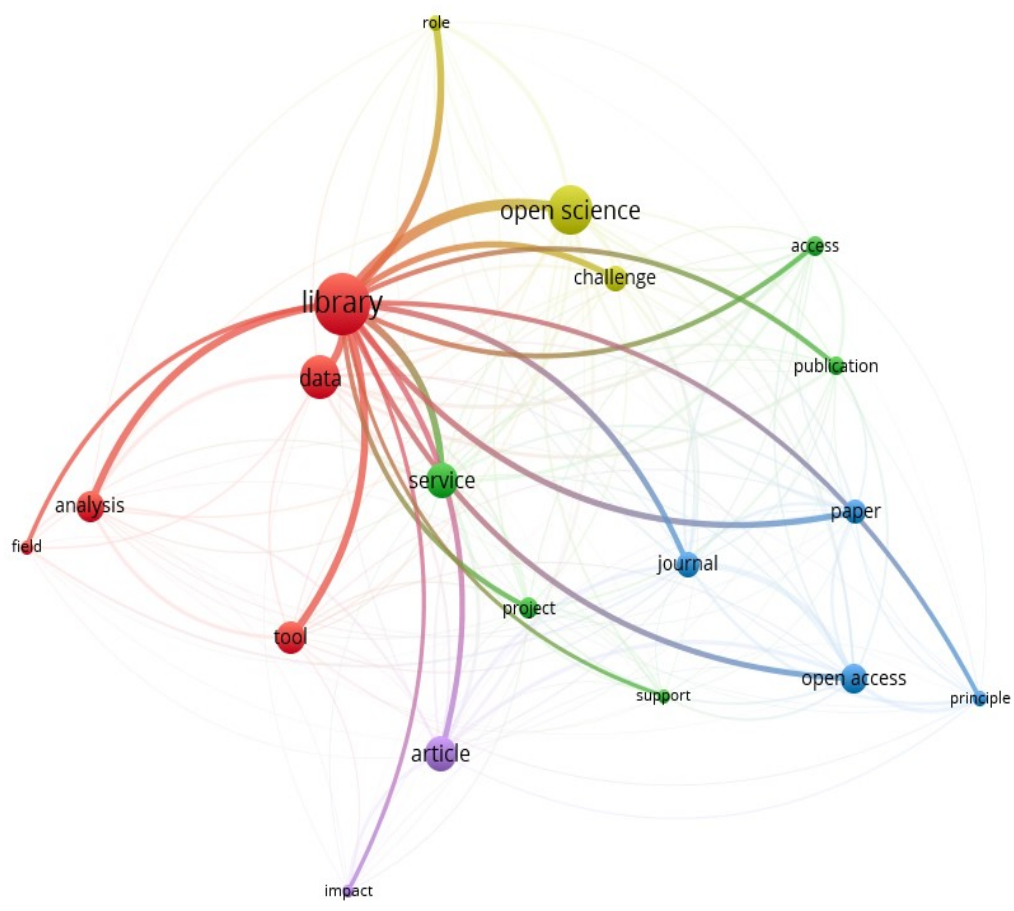

Fuente: VOSViewer

\subsection{Análisis hermenéutico}

3.2.1 Experiencias y retos de trabajo para las bibliotecas académicas frente a la ciencia abierta

A partir de la revisión de la literatura realizada, se presentan los principales hallazgos respecto a los aportes de las bibliotecas académicas. Para esto, se agruparon las temáticas por pilares, los cuales pueden ser vistos como los diferentes frentes de trabajo o retos que deben trabajar estas instituciones para el desarrollo de la ciencia abierta. 
Tabla 1. Principales hallazgos en la literatura del aporte de las bibliotecas académicas a la ciencia abierta.

\begin{tabular}{|c|c|c|}
\hline Pilar & Hallazgos & Trabajos \\
\hline $\begin{array}{l}\text { Políticas y estrategias } \\
\text { para la consolidación } \\
\text { de la ciencia abierta }\end{array}$ & $\begin{array}{l}\text { Bajo este pilar se pueden evidenciar diferentes } \\
\text { trabajos que reflexionan sobre el rol de la biblioteca } \\
\text { académica frente a la ciencia abierta. Principalmente, } \\
\text { se presentan los siguientes hallazgos: } \\
\text { * La colaboración como oportunidad de fortalecer la } \\
\text { ciencia abierta. } \\
\text { * La necesidad de pensar en la creación de } \\
\text { departamentos o unidades para la promoción y } \\
\text { gestión de la ciencia abierta en las universidades. Ante } \\
\text { esto, se destacan los casos de la Universidad de Malta, } \\
\text { donde la biblioteca creó un departamento de ciencia } \\
\text { abierta encargado de la promoción del repositorio } \\
\text { institucional y el diseño de políticas de acceso abierto; } \\
\text { asimismo, para la sensibilización de los investigadores } \\
\text { frente al RI y la ciencia abierta. También se destaca la } \\
\text { experiencia de la Oficina de Conocimiento Abierto } \\
\text { de la Universidad Nacional de Córdoba que, a pesar } \\
\text { de no estar ubicada en la biblioteca, cuenta con la } \\
\text { constante interacción con esta, sobre todo para la } \\
\text { promoción del open access. } \\
\text { * La formación y sensibilización de los } \\
\text { bibliotecólogos frente a la ciencia abierta. }\end{array}$ & $\begin{array}{ll}\circ & \text { Ayris \& Ignat (2018) } \\
\circ & \text { Ayris et al. (2018) } \\
\circ & \text { Ayris et al. (2018) } \\
\circ & \text { Ayris, López de San } \\
& \text { Román, Maes, \& } \\
& \text { Labastida (2018) } \\
\circ & \text { Bueno de la Fuente } \\
& (2016) \\
\circ & \text { Ku \& Bao (2017) } \\
\circ & \text { Maijala (2016) } \\
\circ & \text { Ministerio de } \\
& \text { Educación y Cultura } \\
& \text { de Finlandia (2014) } \\
\circ & \text { Nardi \& Yrusta } \\
& \text { (2014) } \\
\circ & \text { Schöpfel (2017) } \\
\circ & \text { Scicluna (2017) }\end{array}$ \\
\hline $\begin{array}{l}\text { Gestión de datos de } \\
\text { investigación e } \\
\text { investigación } \\
\text { reproducible }\end{array}$ & $\begin{array}{l}\text { A partir de este pilar, se puede ver principalmente la } \\
\text { necesidad de que las bibliotecas académicas apoyen y } \\
\text { sostengan el desarrollo de infraestructuras para la } \\
\text { gestión de datos con miras a facilitar la transparencia } \\
\text { en el ciclo de la investigación y permita el desarrollo } \\
\text { de la investigación reproducible. Asimismo, la } \\
\text { importancia de las estrategias de preservación digital } \\
\text { para la integridad y disponibilidad de los datos. Ante } \\
\text { esto, se pueden ver algunos casos como la } \\
\text { Universidad de Leiden, la Digital Library in High- } \\
\text { Energy Physics del CERN, la Universidad de Viena e } \\
\text { iniciativas del orden europeo. }\end{array}$ & $\begin{array}{ll}\circ & \text { Chen (2016) } \\
\circ & \text { Dallmeier-Tiessen \& } \\
& \text { Mele (2014) } \\
\circ & \text { Gilmore, Adolph \& } \\
& \text { Millman (2016) } \\
\circ & \text { Lyon (2016) } \\
\circ & \text { Parinov (2010) } \\
\circ & \text { Praczyk (2012) } \\
\circ & \text { Raman, Budrone y } \\
& \text { Sánchez Solís (2017) } \\
\circ & \text { Verhaar et al. (2017) }\end{array}$ \\
\hline $\begin{array}{l}\text { Cultura de la ciencia } \\
\text { abierta }\end{array}$ & $\begin{array}{l}\text { Las experiencias y reflexiones sobre ciencia abierta } \\
\text { generan una pregunta recurrente: ¿Cómo promover y } \\
\text { lograr la apropiación de una cultura de la ciencia } \\
\text { abierta? Ante esto, las bibliotecas tienen un rol } \\
\text { fundamental en formación y sensibilización de } \\
\text { investigadores y comunidades académicas. La } \\
\text { literatura muestra la orientación de programas de } \\
\text { alfabetización informacional para la utilización de } \\
\text { repositorios con miras a la investigación reproducible } \\
\text { y la creación e implementación de software educativo } \\
\text { para la formación de públicos. }\end{array}$ & $\begin{array}{ll}\circ & \text { Brewerton (2012) } \\
\circ & \text { Corrado, Moualison } \\
& \text { Sandy \& Mitchell } \\
& (2018) \\
\circ & \text { Hunt y Bakker (2018) } \\
\circ & \text { Lopes, Antunes \& } \\
& \text { Sanches (2018) } \\
& \text { Mitchell, Corrado \& } \\
& \text { Sandy (2018) } \\
\circ & \text { Ogungbeni et al. } \\
& \text { (2018) } \\
\circ & \text { Scicluna (2017) } \\
\circ & \text { Wagner (2018) }\end{array}$ \\
\hline $\begin{array}{l}\text { Acceso abierto, } \\
\text { repositorios digitales }\end{array}$ & $\begin{array}{l}\text { Según los hallazgos de la literatura, este sigue siendo } \\
\text { el pilar de mayor desarrollo en las bibliotecas }\end{array}$ & $\begin{array}{ll}\circ & \text { Ayris }(2017) \\
\circ & \text { Parinov (2010) }\end{array}$ \\
\hline
\end{tabular}




\begin{tabular}{|c|c|c|}
\hline y preservación digital & $\begin{array}{l}\text { académicas. A pesar de que este trabajo no estuvo } \\
\text { enfocado en el acceso abierto, se puede ver que ya } \\
\text { son diversas las reflexiones e iniciativas que se } \\
\text { orientan bajo la perspectiva integral de la ciencia } \\
\text { abierta. Se pueden ver experiencias de la Universidad } \\
\text { Técnica de Bielorrusia, las bibliotecas de la región de } \\
\text { Sub Sahariana (África) y la proyección de los países } \\
\text { europeos y asiáticos. }\end{array}$ & $\begin{array}{ll}\circ & \text { Schneider }(2016) \\
\circ & \text { Seo et al. }(2017) \\
\circ & \text { Siyao et al. }(2017) \\
\circ & \text { Smith \& Rinaldo } \\
& (2016) \\
\circ & \text { van Miert (2016) } \\
\circ & \text { Yurik y Lazarev } \\
& (2018)\end{array}$ \\
\hline $\begin{array}{l}\text { Transformación de la } \\
\text { comunicación } \\
\text { científica }\end{array}$ & $\begin{array}{l}\text { A partir del desarrollo de la ciencia abierta, surgen } \\
\text { múltiples reflexiones sobre el proceso de } \\
\text { transformación de la comunicación científica y el rol } \\
\text { de las bibliotecas académicas. Diversos documentos } \\
\text { hablan de la necesidad de que esta institución } \\
\text { apoye la implementación de herramientas } \\
\text { informáticas para la comunicación, orienten los } \\
\text { procesos de publicación e inclusive, apoyen la } \\
\text { implementación de flujos de evaluación abierta (Open } \\
\text { Peer Review). }\end{array}$ & $\begin{array}{ll}\circ & \text { CARL Scholarly } \\
& \text { Communication } \\
& \text { Roadmap Working } \\
& \text { Group (2017) } \\
\circ & \text { Rath y Wang (2017) } \\
\circ & \text { Revez (2018) } \\
\circ & \text { Tammaro (2017) }\end{array}$ \\
\hline $\begin{array}{l}\text { Servicios para la } \\
\text { ciencia abierta }\end{array}$ & $\begin{array}{l}\text { La revisión de literatura muestra la necesidad de que } \\
\text { las bibliotecas académicas diseñen y fortalezcan } \\
\text { servicios especializados para investigadores, ya no } \\
\text { solo para las primeras etapas de búsqueda de } \\
\text { información, sino que apoye el desarrollo del ciclo de } \\
\text { investigación; facilitando la implementación de } \\
\text { recursos e infraestructuras para ello. De igual modo, } \\
\text { la creación de servicios digitales que rompan con } \\
\text { barreras temporales y espaciales y que de la misma } \\
\text { manera puedan apoyar el desarrollo del ciclo. }\end{array}$ & $\begin{array}{ll}\text { ○ } & \begin{array}{l}\text { Anglada \& Abadal } \\
\text { (2018) }\end{array} \\
\text { ○ } & \text { Grguric, Davis \& } \\
& \text { Davidson (2016) } \\
\circ & \text { Liber Europe (2018) } \\
\circ & \text { Schmidt et al. (2018) } \\
\circ & \text { Schöpfel (2016) }\end{array}$ \\
\hline
\end{tabular}

Fuente: Elaboración propia

Toda esta revisión, evidencia que las bibliotecas académicas juegan un papel fundamental en la promoción de la investigación en todos los niveles, pues estas brindan la posibilidad de ofrecer publicaciones y datos de manera abierta y para uso de todos (WIGBOLDUS; DOTSCH, 2015).

Se puede observar que las principales experiencias se han venido dando en Estados Unidos y Europa (sobre todo por el trabajo de LIBER, ya mencionado, pero también desde LERU-League of European Research Universities) $)^{5}$.

De igual forma, se muestra la receptividad por parte de los investigadores hacia la cooperación con bibliotecólogos para la gestión, formación y explotación de las publicaciones y datos de investigación. Sin embargo, a pesar de todo el esfuerzo que las bibliotecas 
académicas han invertido en apoyo a la ciencia abierta, aún falta mayor visibilidad y masificación en otros contextos para lograr mayor impacto (OGUNGBENI et al, 2015).

\section{Discusión y conclusiones}

Las bibliotecas académicas (universitarias o de investigación), deben involucrarse más en la promoción -y hasta defensa- de la ciencia abierta, ya que es un rol fundamental de las mismas, para responder a esta tendencia imparable, para con ello, prestar servicios de calidad como siempre ha sido su propósito; pero en este momento específico, para alentar a los científicos y a otros agentes del ecosistema de la ciencia abierta, que pueden ser aún desconocedores o escépticos, para unirse a este movimiento, a esta forma de hacer ciencia.

Es decir, como dice Barandiaran, Araya y Vila-Viñas (2015), implica la necesidad de analizar la ciencia abierta no como una nueva creación o tipo de ciencia separada del sistema ya existente, sino la creación de una nueva forma de liberación de los insumos y resultados de la ciencia que se había ido cerrando y privatizando de manera progresiva; es decir, no es una nueva ciencia, es la forma de hacer ciencia el siglo XXI (WATSON, 2015).

Muchas bibliotecas académicas están enfocando toda su atención sobre la construcción y el mantenimiento de repositorios (acceso abierto a las publicaciones, básicamente de artículos y monografías), lo cual es un primer paso considerando todos los componentes de la ciencia abierta, no obstante, es necesario que también incluyan los depósitos de datos y su gestión, para ir un paso más adelante (PESET; GONZÁLEZ, 2017).

Estos dos componentes (acceso abierto y datos abiertos), unidos a la posibilidad de guiar y ofrecer otras métricas, que brinden información complementaria -altmetrics- tanto del impacto científico como del impacto social (con otros públicos: estudiantes, medios de comunicación, políticos, organizaciones, ciudadanía), alentará más a las universidades e instituciones de investigación, a los investigadores mismos; a asumir la ciencia abierta e ir trabajando por los otros componentes: investigación reproducible abierta, herramientas abiertas, etc. y llegar a políticas de ciencia abierta, institucionales y nacionales. Incluso, ir avanzando cada vez más, con acciones específicas, en todos los subcomponentes que detalla la taxonomía del proyecto FOSTER ${ }^{6}$.

${ }^{6}$ https://www.fosteropenscience.eu/foster-taxonomy/open-science (Consultado: 15-7-2018) 
En el caso de las bibliotecas académicas en América Latina, algunas han comenzado a caminar en este sentido y realizar acciones en pro de la ciencia abierta. Un porcentaje importante, también desde el acceso abierto a las publicaciones ${ }^{7}$; pero poco a poco, algunas otras bibliotecas académicas de nuestra región, desde lo que genéricamente denominaríamos "servicios de información y formación para investigadores", están buscando aportar a la investigación (TARANGO; ROMO GONZÁLEZ, 2015), en la forma actual, es decir, como ciencia abierta con todos sus componentes y subcomponentes.

En este sentido, un aspecto importante es poder tener referentes en otros contextos, para con sus debidas adaptaciones y contextualizaciones, poder realizar procesos de implementación de estos servicios, tras realizar procesos de bechmarking de esas experiencias en Estados Unidos, Europa u otros países ${ }^{8}$.

En síntesis, las bibliotecas académicas tienen un papel clave en impulsar la ciencia abierta, desde sus distintas instituciones (lo micro), al ofrecer formación para la apropiación de la ciencia abierta -alfabetización informacional para científicos o data information literacy (CARLSON; JOHNSTON, 2015)- y prestar servicios específicos que apoyen el crecimiento de la ciencia abierta mediante: acceso abierto a las publicaciones, datos abiertos, altmetrics, entre otras ${ }^{9}$; pero también (lo macro), de impulsar políticas de ciencia abierta institucionales (URIBE-TIRADO, 2016) o incluso nacionales (URIBE-TIRADO; OCHOA, 2018), ya que es evidente que desde nuestros contextos, son cada vez más necesarias.

Aún nos falta recorrido, propuestas y acciones en este sentido, por ello para terminar, reiterar ese papel clave de las bibliotecas académicas (universitarias o de investigación) y de los bibliotecólogos, cada vez más formados y dispuestos a estos roles, reconociendo, como dicen Tarango \& Machin-Mastromatteo (2017), que el profesional de la información es la persona

\footnotetext{
7 http://v2.sherpa.ac.uk/view/repository_by_country/019.html (Consultado: 15-7-2018)

${ }^{8}$ Una importante recopilación de la situación en varios países (Alemania, Australia, Brasil, Canadá, Estados Unidos, Holanda, Portugal, Reino Unido y Unión Europea -en general-) se puede conocer en: Livro Verde-Ciência aberta e dados abertos: mapeamento e análise de politicas, infraestruturas e estratégias em perspectiva nacional e internacional (Dos Santos et al, 2017)

9 Para ver algunas propuestas específicas, se puede consultar el trabajo de LOPES, DA LUZ ANTUNES \& SANCHES (2018), donde proponen estas acciones: "Reafirmar a relevância das competências de literacia da informação na comunidade académica; Desenvolver competências dos profissionais das bibliotecas para apoio às atividades de ensino e aprendizagem; Apoiar projetos editoriais de publicaşão académica e científica; Assegurar repositórios institucionais alinhados com os padrões de interoperabilidade e preservação; Criar serviços de apoio à gestão de dados científicos; Potenciar o papel da biblioteca no apoio à investigação, Fomentar parcerias com estruturas de apoio a comunidade académica". O también, se puede consultar el trabajo de diagnóstico de las bibliotecas universitarias españolas de Rey Martín; Camón Luis; Pacheco Olea (2018), que identifican estas acciones, entre otras: "Formación y apoyo en la Utilización de repositorios documentales, Formación y apoyo en la utilización de herramientas open access, Formación y apoyo en temas de derechos de autor, Gestión de currículum, Elaboración de índices bibliométricos, Asesoramiento sobre metodología de citación bibliográfica".
} 
indicada para soportar la producción científica, proveer una guía para desarrollar una cultura científica institucional, es este caso, una cultura científica en pro de la ciencia abierta.

\section{Referencias}

ASSOCIATION OF EUROPEAN RESEARCH LIBRARIES.Introduction Powering Sustainable Knowledge in the Digital Age Three Strategic Directions. 2017. Available at: <https://libereurope.eu/wp-content/uploads/2017/11/LIBER-Strategy-2018-2022.pdf>. Access: 15 jul. 2018.

AIBAR, E. De la ciencia abierta a la investigación abierta: los vínculos entre la producción colaborativa y la cultura científica en la era de Internet. p. 19-31. 2013. In: Fronteras de la ciencia: Hibridaciones. Disponible en <https://www.uoc.edu/webs/eaibar/_resources/documents/Aibar_hibri.pdf>.

Acceso: 15 jul. 2018.

ANGLADA, L.; ABADAL, E. ¿Qué es la ciencia abierta?. Anuario Think EPI, v. 12, p. 292-298, 2018. Disponible en:

<https://recyt.fecyt.es/index.php/ThinkEPI/article/viewFile/thinkepi.2018.43/39164>.

Acceso: 15 jul. 2018.

AYRIS, P. The importance of European collaborations to support Open Science and digital library development. LIBER Quarterly, v. 26, n. 4, p. 244, 2017. Available at: <https://doi.org/10.18352/lq.10147>. Cited: 15 jul. 2018.

AYRIS, P.; IGNAT, T. Defining the role of libraries in the Open Science landscape: a reflection on current European practice. Open Information Science, v. 2, n. 1, p. 1-22._2018. Available at: <https://doi.org/10.1515/opis-2018-0001>. Cited: 15 jul. 2018.

AYRIS, P. ET AL. LIBER Open Science Roadmap. 2018. Available at: <https://digital.csic.es/handle/10261/167474>. Cited: 15 jul. 2018.

AYRIS, P. ET AL. Open Science and its role in universities: a roadmap for cultural change. League of European Research Universities. 2018. Available at: <https://www.leru.org/publications/openscience-and-its-role-in-universities-a-roadmap-for-cultural-change>. Cited: 15 jul. 2018.

BARTLING, S.; FRIESIKE, S. (Eds.). Opening Science. Cham: Springer International Publishing. p. 17-47. Available at: <https://doi.org/10.1007/978-3-319-00026-8_2 >. Cited: 15 jul. 2018.

BREWERTON, A. Re-skilling for research: investigating the needs of researchers and how library staff can best support them. New Review of Academic Librarianship, v. 18, n. 1, p. 96-110, 2012. Available at:: <https://doi.org/10.1080/13614533.2012.665718>. Cited: 15 jul. 2018.

BUENO DE LA FUENTE, G. Libraries: roles and opportunities on Open Science. FOSTER, 2016. Available at: <https://www.fosteropenscience.eu/content/libraries-roles-and-opportunities-openscience>. Cited: 15 jul. 2018.

CARL Scholarly Communication Roadmap Working Group. Scholarly Communications Roadmap. 2017. Available at: <http://www.carl-abrc.ca/wpcontent/uploads/2017/03/CARL_ScholComm_Roadmap_EN.pdf>. Cited: 15 jul. 2018. 
CARLSON, J.; JOHNSTON, L. R. (Eds.). Data information literacy: Librarians, data, and the education of a new generation of researchers. Purdue University Press. V. 2, 2015. 272p.

CHEN, X.. ET AL . CERN Analysis Preservation: A Novel Digital Library Service to Enable Reusable and Reproducible Research. In: FUHR, N.. ET AL (Eds.), Research and Advanced Technology for Digital Libraries, v. 9819, p. 347-356, 2016. Cham: Springer International Publishing. Available at: <https://doi.org/10.1007/978-3-319-43997-6_27>._Cited: 15 jul. 2018.

CORRADO, E. M.; MOUALISON SANDY, H.; MITCHELL, E. T. Nullis in Verba: The Free Software Movement as a model for Openness and Transparency. Technical Services Quarterly, v. 35, n. 3, p. 269-279, 2018. Available at: <https://doi.org/10.1080/07317131.2018.1456849>. Cited: 29 jun. 2018.

CROUZIER, T. Science Ecosystem 2.0: how will change occur. Luxembourg: Publications Office of the European Union, 2015. Available at: <http://biopolymersforlife.org/wpcontent/uploads/2015/11/science_ecosystem_2.0-how_will_change_occur_crouzier_072015.pdf > . Cited: 29 jun. 2018.

DALLMEIER-TIESSEN, S.; MELE, S. Integrating data in the scholarly record: Community-driven digital libraries in high-energy physics. Zeitschrift Fur Bibliothekswesen Und Bibliographie, v. 61, n. 4-5, p.220-223, 2014. Available at: <https://zs.thulb.unijena.de/receive/jportal_jparticle_00324882>.Cited: 29 jun. 2018.

DALLMEIER-TIESSEN, S., ET AL. Integrating scholarly publications and research data-preparing for open science, a case study from high-energy physics with special emphasis on (Meta) data models. Research Conference on Metadata and Semantic Research, Springer, Berlin, Heidelberg. p. 146157, 2012). Available at: < https://www.springerprofessional.de/en/integrating-scholarly-publicationsand-research-data-preparing-f/4029378>. Cited: 29 jun. 2018.

DAVID, P. A. Understanding the emergence of «open science» institutions: functionalist economics in historical context. Industrial and Corporate Change, v. 13, n. 4, p. 571-589, 2004. Available at: <https://doi.org/10.1093/icc/dth023>. Cited: 29 jun. 2018.

DOS SANTOS, P. X. ET AL. Livro Verde-Ciência aberta e dados abertos: mapeamento e análise de políticas, infraestruturas e estratégias em perspectiva nacional e internacional. 2017. Disponível em: $<$ https://www.arca.fiocruz.br/bitstream/icict/24117/2/Livro-Verde-07-06-2018.pdf >. Acesso em: 29 jun. 2018.

FECHER, B.; FRIESIKE, S. Open Science: One Term, Five Schools of Thought. Opening science, Springer, Cham.p. 17-47, 2014.

FOSTER. Open Science Taxonomy. 2015. Available at: <https://www.FOSTERopenscience.eu/FOSTER-taxonomy/open-science>. Cited: 29 jun. 2018.

GILMORE, R. O.; ADOLPH, K. E.; MILLMAN, D. S. Curating identifiable data for sharing: The databrary project. In: IEEE, p. 1-6, 2016. Anais... Available at: <https://doi.org/10.1109/NYSDS.2016.7747817> Cited: 29 jun. 2018.

GRGURIC, E.; DAVIS, H.; DAVIDSON, B. Supporting the modern research workflow: Supporting the Modern Research Workflow. Proceedings of the Association for Information Science and Technology, v. 53, n. 1, p. 1-4, 2016. Available at: <https://doi.org/10.1002/pra2.2016.14505301136>._Cited: 29 jun. 2018. 
HIGGINS, J. P. T.; GREEN, S. (eds.). Manual Cochrane de revisiones sistemáticas de intervenciones. The Cochrane Collaboration. 2011. Available at: <http://handbook.cochrane.org>. Cited: 29 jun. 2018.

HUNT, S. L.; BAKKER, C. J. A qualitative analysis of the information science needs of public health researchers in an academic setting. Journal of the Medical Library Association, v. 106, n. 2, 2018. Available at: <https://doi.org/10.5195/JMLA.2018.316>. Cited: 29 jun. 2018.

JAGUSZEWSKI, J. M.; WILLIAMS, K. New Roles for New Times: Transforming Laison Roles in Research Libraries (Report prepared for the Association of Research Libraries). 2013. Available at: $<$ http://www.arl.org/storage/documents/publications/nrnt-liaison-roles-revised.pdf > . Cited: 29 jun. 2018.

KU, L. P.; BAO, Q. The Open Search. Org in Open Science Era: A Communication Platform for Everyone Building Their Repositories and Using Others. In: Advanced Applied Informatics, IIAIAAI, 6, p. 124-128, 2017. Anais... Available at: <https://doi.org/10.1109/IIAI-AAI.2017.221>. Cited: 29 jun. 2018.

LIBER EUROPE. Research Libraries Powering Sustainable Knowledge in the Digital. 2018. Available at: <https://libereurope.eu/wp-content/uploads/2016/11/Nov2016Mail_Draft-StrategicFramework-2022.pdf>. Cited: 29 jun. 2018.

LOPES, C. A.; DA LUZ ANTUNES, M.; SANCHES, T. La contribución de la alfabetización informacional a la Ciencia Abierta. Ibersid: revista de sistemas de información y documentación, v. 12, n. 1, p. 59-67, 2018. Disponible en:

<https://www.ibersid.eu/ojs/index.php/ibersid/article/view/4437>. Acceso en: 29 jun. 2018.

NOVA LYON, L. Transparency: The Emerging Third Dimension of Open Science and Open Data. LIBER Quarterly, v. 25, n. 4, p. 153-171, 2016. Available at:: < https://doi.org/10.18352/lq.10113>. Cited: 29 jun. 2018.

MAIJALA, R. Joining Networks in the World of Open Science. LIBER Quarterly, v. 26, n. 3, p. 104124, 2016. Available at: <https://doi.org/10.18352/lq.10179>. Cited: 29 jun. 2018.

MINISTERIO DE EDUCACIÓN Y CULTURA DE FINLANDIA. Open science and research lead to surprising discoveries and creative insights: Open science and research roadmap 2014 2017. 2014. Available at:

$<$ https:// openscience.fi/documents/14273/0/Open+Science+and+Research+Roadmap+20142017/e8eb7704-8ea7-48bb-92e6-c6c954d4a2f2>. Cited: 29 jun. 2018.

MITCHELL, E. T.; CORRADO, E. M.; SANDY, H. M. Nullis in Verba: Advancing Research through Transparency and Openness. International Information \& Library Review, v. 50, n. 2, p. 157-162, 2018. Available at: <https://doi.org/10.1080/10572317.2018.1449432>. Cited: 29 jun. 2018.

NARDI, A.; YRUSTA, L. Ciencia Abierta y los desafíos de la Oficina Conocimiento Abierto de la Universidad Nacional de Córdoba. In: Jornada sobre la Biblioteca Digital Universitaria, JBDU, 12, Salta, 2014. Anais... Disponible en: <http://eprints.rclis.org/24170/> Acceso em: 29 jun. 2018.

OCDE. Open science country notes. 2015. Available at:

$<$ https://www.innovationpolicyplatform.org/content/open-science-country-notes>.

Cited: 29 jun. 2018. 
OGUNGBENI, J. I. ET AL. The roles of academic libraries in propagating open science: a qualitative literature review. Information Development, v. 34, n. 2, p. 113-121, 2018. Available at: <https://doi.org/10.1177/0266666916678444>. Cited: 29 jun. 2018.

PARINOV, S. The electronic library: using technology to measure and support Open Science. In: Proceedings of the World Library and Information Congress, 76, Gothenburg, Sweden, p. 10-15, 2010. Available at: <https://www.ifla.org/past-wlic/2010/155-parinov-en.pdf>. Cited: 29 jun. 2018.

PÉREZ, J. Revisión sistemática de literatura en ingeniería. 2012. Universidad de Antioquia, Medellín.

PESET, F.; GONZÁLEZ, L. M. Ciencia abierta y gestión de datos de investigación. Gijón: TREA, 2016. 192p.

PRACZYK, P. ET AL. Integrating scholarly publications and research data-preparing for open science, a case study fromhigh-energy physics with special emphasis on (Meta) data models. In: Research Conference on Metadata and Semantic Research, p. 146-157. Springer, Berlin, Heidelberg2012. Anais... Available at: < https://www.springerprofessional.de/en/integratingscholarly-publications-and-research-data-preparing-f/4029378> . Cited: 29 jun. 2018.

RAMAN, R.; BUDRONE, P.; SÁNCHEZ SOLÍS, B. Living Digital Ecosystems for Data Preservation: An Austrian Use Case Towards the European Open Science Cloud. Stand Alone, p. 203-210, 2017. Available at:: <https://doi.org/10.3233/978-1-61499-769-6-203>. Cited: 29 jun. 2018.

RATH, M.; WANG, P. Open peer review in the era of open science: a pilot study of Researchers' perceptions. In: ACM/IEEE, 17, p. 317-318, 2017. Anais... Available at: <https:// dl.acm.org/citation.cfm?id=3200334.3200391>. Cited: 29 jun. 2018.

REILLY, S. Libraries Enabling Open Science: LIBER Strategy \& Advocacy. In: LAI/CILIP, Ireland, 2016. Anais... Available at: < https://www.slideshare.net/libereurope/libraries-enabling-open-scienceliber-strategy-advocacy>. Cited: 29 jun. 2018.

REVEZ, J. Opening the Heart of Science: A Review of the Changing Roles of Research Libraries. Publications, v. 6, n. 1, p. 9, 2018. Available at: <https://doi.org/10.3390/publications6010009> Cited: 29 jun. 2018.

REY MARTÍN, C.; CAMÓN LUIS, E.; PACHECO OLEA, F. El Soporte a la investigación en las bibliotecas universitarias españolas. Anales de Documentación, v. 21, n. 1, 2018. Disponible en: <http://revistas.um.es/analesdoc/article/view/295841/224511>. Acceso en: 29 jun. 2018.

SALVADOR-OLIVÁN, J. A.; MARCO-CUENCA, G.; ARQUERO-AVILÉS, R. Las revisiones sistemáticas en Biblioteconomía y Documentación: análisis y evaluación del proceso de búsqueda. Revista Española de Documentación Científica, v. 41, n. 2, p. 207, 2018. Disponble en: <https://doi.org/10.3989/redc.2018.2.1491>. Acceso em: 29 jun. 2018.

SCHMIDT, B. Open Science Support as a Portfolio of Services and Projects: From Awareness to Engagement. Publications, v. 6, n. 2, p. 27, 2018. Available at: <https://doi.org/10.3390/publications6020027>. Cited: 29 jun. 2018.

SCHNEIDER, G. Scientific Information: Access, Processing and Safeguarding. A Program Combining Efforts to Manage Scientific Information in Switzerland. Bibliothek Forschung und Praxis, v. 40, n. 3, 2016. Available at: <https://doi.org/10.1515/bfp-2016-0069>. Cited: 29 jun. 2018. 
SCHÖPFEL, J. Open supply? On the future of document supply in the world of open science.

Interlending \& Document Supply, v. 44, n. 4, p. 150-154, 2016. Available at:

$<$ https://doi.org/10.1108/ILDS-07-2016-0025>. Cited: 29 jun. 2018.

SCHÖPFEL, J. Six Futures of Academic Libraries. In: The End of Wisdom? Elsevier, p. 123-128, 2017. Available at: <https://doi.org/10.1016/B978-0-08-100142-4.00013-0>. Cited: 29 jun. 2018.

SCICLUNA, R. Open Access Marketing at the University of Malta: A case study of how the University of Malta Library set up The Open Science Department to help market and promote the Open Access institutional repository-OAR@UoM. 2017. Available at:

<http://library.ifla.org/1887/1/S12-2017-scicluna-en.pdf>. Cited: 29 jun. 2018.

SEO, J.-W. ET AL. Equality, equity, and reality of open access on scholarly information. Science Editing, v, 4, n. 2, p. 58-69, 2017. Available at: <https://doi.org/10.6087/kcse.97>. Cited: 29 jun. 2018.

SIYAO, P. O. ET AL. Academic libraries in four Sub-Saharan Africa countries and their role in propagating open science. IFLA Journal, v. 43, n. 3, p. 242-255, 2017. Available at: $<$ https://doi.org/10.1177/0340035217712263>. Cited: 29 jun. 2018.

SMITH C.; MARSAN G. A. Open Science: The policy challenges - The open science ecosystem: a complex picture. 2015. Available at: < http://www.chairedelimmateriel.u-psud.fr/wpcontent/uploads/2016/09/Dominique-Guellec.pdf>. Cited: 29 jun. 2018.

SMITH, J. E.; RINALDO, C. A. Collaborating on open science: The journey of the Biodiversity Heritage Library. Information Services \& Use, v. 35, n. 4, p. 211-216, 2016. Available at: $<$ https:// doi.org/10.3233/ISU-150785>. Cited: 29 jun. 2018.

TAMMARO, A. M. Digital Scholarship Innovation and Digital Libraries: A Survey in Italy. In: AGOSTI, M. ET AL (Eds.). Digital Libraries and Multimedia Archives. V. 701. Cham: Springer International Publishing, p. 31-43, 2017. Available at: <https://doi.org/10.1007/978-3-319-563008_4>. Cited: 29 jun. 2018.

TARANGO, J.; ROMO-GONZÁLEZ, J. R. Bibliotecas académicas e investigación. Buenos Aires: Alfagrama Ediciones, 2015. 208p.

TARANGO, J.; MACHIN-MASTROMATTEO, J. D. The Role of Information Professionals in the Knowledge Economy: Skills, Profile and a Model for Supporting Scientific Production and Communication. USA: Chandos Publishing, 2017.136 p.

URIBE-TIRADO, A. La Universidad de Antioquia, debe ser una universidad de Ciencia Abierta. Portal: Universidad de Antioquia: 2016. Disponível em: <http://eprints.rclis.org/30174/ Cited: 29 jun. 2018.

URIBE TIRADO, A.; OCHOA, J. Perspectivas de la ciencia abierta: un estado de la cuestión para una política nacional en Colombia. BiD: textos universitaris de biblioteconomia i documentación, $\mathrm{n}$. 40, 2018. Disponible en: <http://dx.doi.org/10.1344/BiD2018.40.5>. Acceso: 29 jun. 2018.

VAN MIERT, D. A Conceptual Approach to Library History: Towards a History of Open Science. Quaerendo, v. 46, n. 2-3, p. 205-221, 2016. Available at: <https:// doi.org/10.1163/1570069012341355>. Cited: 29 jun. 2018. 
VERHAAR, P. ET AL. Fostering Effective Data Management Practices at Leiden University. LIBER Quarterly, v. 27, n. 1, p. 1-22, 2017. Available at: <https://doi.org/10.18352/lq.10185>. Cited: 29 jun. 2018.

VICENTE-SAEZ, R.; MARTINEZ-FUENTES, C. Open Science now: A systematic literature review for an integrated definition. Journal of Business Research, v. 88, p. 428-436, 2018. Available at: <https://doi.org/10.1016/j.jbusres.2017.12.043>. Cited: 29 jun. 2018.

WAGNER, G. Sim4edu.com - Web-based simulation for education. In: Simulation Conference, 2018. Anais... Available at: <https://doi.org/10.1109/WSC.2017.8248130>. Cited: 29 jun. 2018.

WATSON, M. When will 'open science' become simply 'science'?. Genome biology, v. 16, n. 1, p. 1, 2015. Available at: <http://genomebiology.biomedcentral.com/articles/10.1186/s13059-015-0669-2>. Cited: 29 jun. 2018.

WIGBOLDUS, D.H.J.; DOTSCH, R. Psychometrika. 2016. 27p. Available at: <https:// doi.org/10.1007/s11336-015-9445-1>. Cited: 29 jun. 2018.

YURIK, I., \& LAZAREV, V. Open science: principles in the activities of a university library. 2018. Available at: <https://rep.bntu.by/handle/data/41913>. Cited: 29 jun. 2018.

Data de submissão: 03-08-2018

Data de aceitação: 01-09-2018 